\title{
Toxoplasma gondii seroprevalence in wild boars (Sus scrofa) in Sweden and evaluation of ELISA test performance
}

\author{
C. WALLANDER ${ }^{1 *}$, J. FRÖSSLING ${ }^{2,3}$, I. VÅGSHOLM ${ }^{1}$, A. UGGLA $^{1}$ AND \\ A. LUNDÉN ${ }^{1,4}$ \\ ${ }^{1}$ Department of Biomedical Sciences and Veterinary Public Health, Swedish University of Agricultural Sciences, \\ Uppsala, Sweden \\ ${ }^{2}$ Department of Disease Control and Epidemiology, National Veterinary Institute, Uppsala, Sweden \\ ${ }^{3}$ Department of Animal Environment and Health, Swedish University of Agricultural Sciences, Skara, Sweden \\ ${ }^{4}$ Department of Virology, Immunobiology and Parasitology, National Veterinary Institute, Uppsala, Sweden
}

Received 27 January 2014; Final revision 24 September 2014; Accepted 9 October 2014; first published online 6 November 2014

\section{SUMMARY}

Toxoplasma gondii is a zoonotic protozoan parasite, infecting a wide range of warm-blooded animals. The Swedish wild boar population is expanding and increased hunting provides its meat to a growing group of consumers. We performed a spatio-temporal investigation of $T$. gondii seroprevalence in Swedish wild boars. An ELISA was set up and evaluated against a commercial direct agglutination test, using Bayesian latent class analysis. The ELISA sensitivity and specificity were estimated to $79 \%$ and $85 \%$, respectively. Of 1327 serum samples, $50 \%$ were positive. Thirty-four per cent of young wild boars and $55 \%$ of adults were positive $(P<0.001)$. The total seroprevalence ranged from $72 \%$ in 2005 to $38 \%$ in $2011(P<0 \cdot 001)$, suggesting a declining trend. The highest seroprevalence, $65 \%$, was recorded in South Sweden. In other regions it varied from $29 \%$ in Stockholm to $46 \%$ in East Middle Sweden.

Key words: Monitoring, serological screening, test evaluation, Toxoplasma gondii, wild boar.

\section{INTRODUCTION}

Toxoplasma gondii is a zoonotic, potentially lethal, protozoan parasite with the ability to infect most warm-blooded animals, including humans. Infection occurs by ingesting oocysts, excreted into the environment with the faeces from newly infected cats (the main host of the parasite) or by consuming meat from previously infected intermediate host animals harbouring tissue cysts [1]. Recently, several studies have estimated $T$. gondii to be one of the most

\footnotetext{
* Author for correspondence: Ms. C. Wallander, Swedish University of Agricultural Sciences, Department of Biomedical Sciences and Veterinary Public Health, Box 7036, 75007 Uppsala, Sweden.

(Email: camilla.gustafsson@slu.se)
}

important pathogens involved in foodborne infections, causing a considerable disease burden and economic losses [2, 3]. The European Food Safety Authority (EFSA) has recommended the implementation of pre-harvest monitoring of $T$. gondii in sheep, goats, pigs, and game [4]. In Sweden, studies have been performed on $T$. gondii prevalence in different wildlife species such as brown hares (Lepus europaeus P.) [5], red foxes (Vulpes vulpes) [6], moose (Alces alces), roe deer (Capreolus capreolus) [7] and lynx (Lynx lynx) [8]. Wild boars (Sus scrofa) are located mainly in the southeastern part of the country but are, according to the Swedish Association for Hunting and Wildlife Management, rapidly and successively spreading north [9]. The destructive behaviour of wild boars in agricultural areas has 
demanded increased hunting and, as a result, wild boar meat is now available for a larger group of consumers. The prevalence of $T$. gondii in wild boars in Sweden has not been previously investigated. However, in nearby parts of Europe, studies have indicated seroprevalence rates at high levels, ranging from $25 \%$ to $35 \%$ [10-12]. Two of the most widely used methods for seroprevalence studies of $T$. gondii are direct agglutination tests (DAT) and enzyme-linked immunosorbent assays (ELISA) [13], the latter being practical and comparatively inexpensive if set up as an in-house system. It is essential that new tests are validated against a gold standard test and/or using sera from a population of animals with known disease status. However, test evaluation is also possible using, for example, a Bayesian statistical approach [14], which has been used in veterinary diagnostic test evaluations in recent years (e.g. see [15] and [16]). To assess the importance of wild boar meat as a source of $T$. gondii infection, the aims of this study were to: $(a)$ evaluate the test performance of an in-house ELISA against a commercially available DAT, $(b)$ estimate the prevalence of T. gondii antibodies in wild boars in Sweden based on submitted samples, and $(c)$ investigate possible variations in seroprevalence due to age, region and year.

\section{METHODS}

\section{Background population and study sample}

The wild boar population in Sweden increased to more than 100000 wild boars by $2010 / 2011$ (July-June) compared to 40000 animals in 2005/ 2006 [9]. The geographical distribution of wild boars has expanded during the study period from the southern area to include the middle-eastern parts of the country. The population distribution for 2009/2010 and 2010/2011 is shown in Figure 1. The material used in this study is a subset of samples from a national surveillance programme for absent diseases in Swedish wild boars [17]. This programme has been on-going since 2000 and is based on hunters submitting wild boar serum samples to the National Veterinary Institute (SVA) in Uppsala, Sweden. Information on geographical origin is available for most samples, and for samples collected in 2010 and onwards, information on age group based on phenotype (colour and size) is also registered. In this study the age groups were $\leqslant 12$ months and $>12$ months.

The samples analysed in this study were retrieved from samples collected in 2005 and from 2008 to 2011.

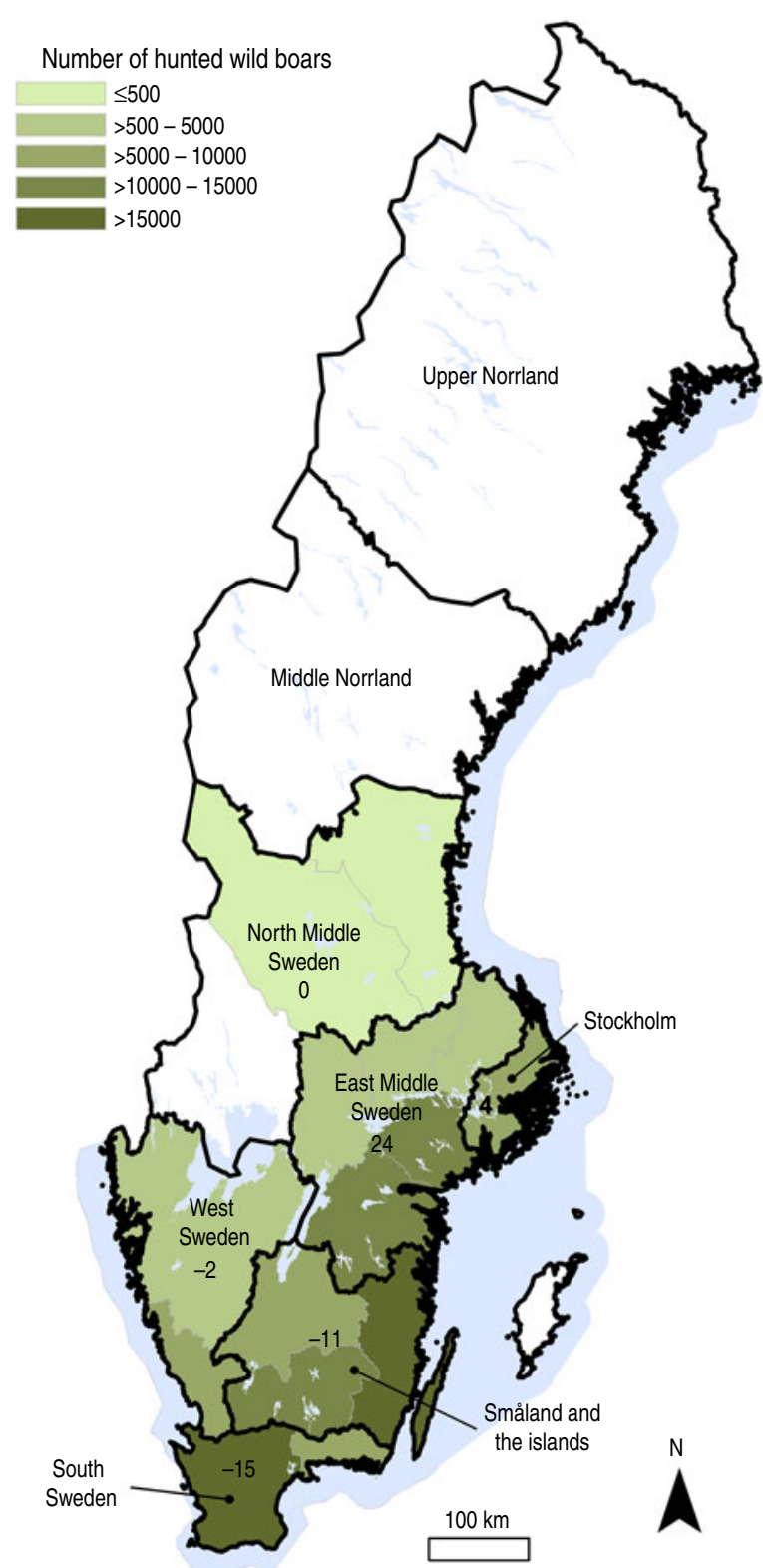

Fig. 1. Geographical distribution of hunted wild boars in Sweden during the hunting seasons of 2009/2010 and 2010/2011. Based on data provided by the Swedish Association for Hunting and Wildlife Management, Wildlife Monitoring [9]. Colour scale represents number of animals hunted in each county (white areas represent no wild boars hunted). Regions (Nomenclature of Territorial Units for Statistics level 2) are drawn with black borders and black numbers on the map indicate percentage difference between proportion of hunted animals and proportion of sampled animals in each region. For example, South Sweden is underrepresented in our sample compared to the proportion of wild boars hunted in this region.

The strategy was to randomly select 250 samples from each year. This sample size was based on a population size of 100000-150000 wild boars, a maximum 
Table 1. Number and distribution of serum samples per year, age group (in months) and region

\begin{tabular}{|c|c|c|c|c|c|c|c|}
\hline Variable & Category & 2005 & 2008 & 2009 & 2010 & 2011 & Total \\
\hline \multirow[t]{4}{*}{ Age } & $>12$ & n.a. & n.a. & n.a. & 106 & 99 & 205 \\
\hline & $\leqslant 12$ & n.a. & n.a. & n.a. & 132 & 143 & 275 \\
\hline & Unknown & n.a. & n.a. & n.a. & 12 & 28 & 40 \\
\hline & Total & n.a. & n.a. & n.a. & 250 & 270 & 520 \\
\hline \multirow{8}{*}{ Region } & South Sweden & 46 & 20 & 47 & $43(23 / 18)^{*}$ & $12(8 / 3)^{*}$ & 168 \\
\hline & Småland and the Islands & 19 & 121 & 63 & $45(25 / 20)^{*}$ & $51(21 / 27)^{*}$ & 299 \\
\hline & West Sweden & 33 & 105 & 43 & $27(16 / 10)^{*}$ & $18(17 / 1)^{*}$ & 226 \\
\hline & East Middle Sweden & 67 & 118 & 87 & $107(46 / 54)^{*}$ & $144(70 / 60)^{*}$ & 523 \\
\hline & Stockholm & 3 & 5 & 8 & $27(21 / 4)^{*}$ & $28(22 / 5)^{*}$ & 71 \\
\hline & North Middle Sweden & 12 & 0 & 0 & $1(1 / 0)^{*}$ & 0 & 13 \\
\hline & Unknown & 10 & 0 & 0 & 0 & $17(5 / 3)^{*}$ & 27 \\
\hline & Total & 190 & 369 & 248 & 250 & 270 & 1327 \\
\hline
\end{tabular}

n.a., Not applicable.

* Total number ( $\leqslant 12$ months/>12 months).

expected seroprevalence of $25 \%$ [10-12] and an aim to detect a 10\% change in seroprevalence with $95 \%$ confidence level and 80\% power (Win Episcope $2 \cdot 0$ [18]). Between $10 \%$ and $30 \%$ of the samples were excluded due to poor quality; moreover, only a limited number of samples were available from 2005 and 2009. Finally, 1328 samples were included in the study and these represented six regions in Sweden (Nomenclature of Territorial Units for Statistics (NUTS) system, level 2 [19]) (Fig. 1). Information on animal age was available for 480 samples. The distribution of samples per year, age group and region, is shown in Table 1.

\section{Serology}

\section{ELISA}

All serum samples were analysed using ELISA. This was based on a previously published assay for sheep sera utilizing a saponin-octylglucoside solubilized tachyzoite antigen from the RH strain of $T$. gondii, produced at the Moredun Research Institute, UK. The assay was performed as described previously [20] but with a peroxidase-labelled polyclonal rabbit anti-pig immunoglobulin $\mathrm{G}$ antibody (Sigma-Aldrich, USA) diluted 1: 10000 , Polysorp microtitre plates (Nunc Immuno Plate, Thermo Fisher Scientific, USA) and buffers and substrate from SVA. Washing was performed with a manual plate washer (Nunc-Immuno Wash 12, Thermo Fisher Scientific) and the optical density (OD) at $450 \mathrm{~nm}$ was read in a plate reader (Multiscan FC v. 2.5, Thermo Fisher Scientific). Positive and negative pig sera were used in checkerboard titrations to retrieve optimal dilutions for antigen, serum samples and conjugate, for which the ratio between OD values for positive and negative sera was as high as possible. The positive sera was provided by the National Veterinary Institute, Technical University of Denmark (Kgs. Lyngby, Denmark), and the negative sera were from fattening pigs in a closed herd (Serogrisen, Ransta, Sweden) which tested negative by DAT (described below) and immunoblotting, performed as described previously [21]. Control for plate-to-plate variation was performed as described previously [20], using seven controls, ranging in OD value from $0 \cdot 10$ to $1 \cdot 52$. A total of four ELISA plates were retested based on the calculated $R^{2}(<0 \cdot 97)$ [20]. Further, 25 individual samples were retested because of a coefficient of variation $(\mathrm{CV}$; i.e. standard deviation of samples/mean value of samples) $>20 \%$ between the duplicates. One sample did not manage to obtain a $\mathrm{CV}<20 \%$ in spite of multiple analyses and was excluded from the dataset.

\section{$D A T$}

To allow estimation of diagnostic sensitivity (Se) and specificity $(\mathrm{Sp})$ of the ELISA on wild boar serum samples, a subset of the samples $(n=242)$ from 2011 was also analysed using a commercially available DAT, in which IgM-mediated agglutination is prevented by addition of 2-mercaptoethanol (Toxo-Screen DA, bioMérieux SA, France). The DAT was performed according to the manufacturer's instructions except that a serum dilution of 1:20 was used as positive cut-off. This cut-off has previously been evaluated for pig sera [22]. Samples were also tested in dilution 1:4000 to account for the prozone effect [23]. Sera positive in either dilution were classified as $T$. gondii positive. Out of 89 
DAT-positive samples, 24 were positive only in dilution 1:4000. Seventy per cent of these samples had ELISA OD values $>0 \cdot 8$, which agrees well with the prozone effect being a result of high levels of specific antibodies.

\section{Estimation of diagnostic Se and Sp}

The test performance of the ELISA was evaluated using Bayesian latent class analysis based on two dependent tests and two populations as described by Branscum et al. [24]. For evaluation purposes, a subset of samples from $2011(n=242)$ were grouped into subpopulation 1 [wild boars aged $\leqslant 12$ months $(n=143)$ ] and subpopulation 2 [wild boars aged $>12$ months $(n=99)]$. Based on numerous reports of an age-dependent $T$. gondii prevalence in a wide range of host species [11, 12], the prevalence in subpopulation $1(\pi 1)$, was expected to be lower compared to the prevalence of subpopulation $2(\pi 2)$. The latent class model was run using OpenBUGS 3.2.2 [25]. After a burn-in period of 5000 iterations, estimates were based on the next 50000 iterations. Multiple chains (set to different starting values) for every estimate were checked for convergence and stability by assessing history plots [26]. Prior information on the parameters to be estimated was included in the model as beta distributions. The priors for the diagnostic Se and $\mathrm{Sp}$ of DAT ( $\mathrm{Se}_{\mathrm{DAT}} / \mathrm{Sp}_{\mathrm{DAT}}$ ) were set to a mode and 5th percentile of 0.83 and 0.65 , and 0.90 and $0 \cdot 80$, respectively. This corresponded to beta $(17 \cdot 86,4 \cdot 45)$ and beta $(42 \cdot 57,5 \cdot 62)$ and was based on previously published evaluations of the test $[22,24]$. To get a rough prior estimate of the seroprevalence in the young and adult population $(\pi 1 / \pi 2)$ a commercial ELISA (ID Screen Toxoplasmosis Indirect Multi-species; IDvet Innovative Diagnostics, France) was used. The apparent seroprevalence was $27 \%$ (95th percentile $=35 \%$ ) in the young population and $40 \% \quad(95$ th percentile $=51 \%)$ in the adult population. Using these estimates, and the 95 th percentiles in the confidence intervals as the 95th percentiles for the beta distributions, provided beta $(27 \cdot 57,72 \cdot 85)$ and beta(23.60,34.91) as priors for $\pi 1 / \pi 2$, respectively. For Se analysis purposes, the model was also run using uniform priors [beta $(1,1)]$ for both DAT test performance and prevalences. A series of cut-off-values for the ELISA were tested in the analysis to identify a suitable cut-off for our purpose. The selected ELISA cut-off value was based on the results from the latent class analysis and with regard to the calculated maximal Youden's index

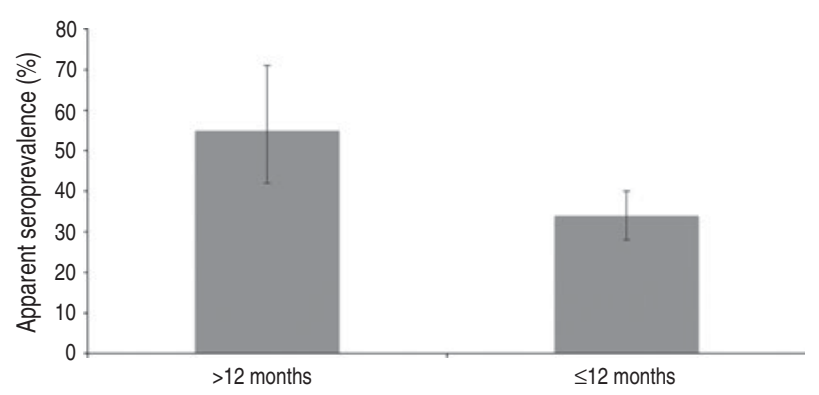

Fig. 2. Apparent seroprevalence (with $95 \%$ confidence intervals) in animals aged $\leqslant 12$ months and $>12$ months in 2010-2012.

(Youden's index $=\mathrm{Se}+\mathrm{Sp}-1$ ) [27]. Using this cut-off, all 1327 samples were classified as positive or negative.

Data management and statistical analysis was performed using $\mathrm{R} \quad 2 \cdot 15 \cdot 2$ [28]. Differences between groups were tested using the Pearson's $\chi^{2}$ test.

\section{RESULTS}

\section{Seroprevalence in wild boars}

After classifying samples as positive or negative using the selected cut-off (see below), 657/1327 samples [50\%; 95\% confidence interval (CI) 47-52] scored positive. In total, 94/275 (34\%, 95\% CI 29-40) young wild boars and 113/205 (55\%, 95\% CI 48-62) adult wild boars were positive (Fig. 2). The difference in seroprevalence between the two age groups was significant $(P<0 \cdot 001)$. The apparent seroprevalence per year varied between $38 \%(95 \%$ CI 32-44) in 2011 and $72 \%(95 \%$ CI $65-78)$ in 2005 (Fig. 3). The difference was significant between 2005 and $2011(P<0 \cdot 001)$ and also between 2008 (50\%, 95\% CI 45-55) and 2011 $(P<0 \cdot 05)$. Based on pair-wise comparisons, the apparent seroprevalence within different regions (considering only years 2010 and 2011 together, due to the rapidly changing population) was similar except for South Sweden, which had a significantly higher seroprevalence than all other regions $(65 \%, 95 \%$ CI $51-77)$ $(P<0.05)$ (Fig. 4). The seroprevalence in other regions varied from $29 \%$ (95\% CI 18-43) in Stockholm to 46\% (95\% CI 40-52) in East Middle Sweden.

\section{Test performance}

The estimated $\mathrm{Se}$ and $\mathrm{Sp}$ of the ELISA at different cut-offs, based on the latent class analysis, are presented in Figure 5. Of all cut-offs tested $(0 \cdot 30-0 \cdot 60)$, cut-off values $0 \cdot 35-0 \cdot 41$ showed the highest combined values for $\mathrm{Se}$ and $\mathrm{Sp}(\mathrm{Se}=80-78 \%, \mathrm{Sp}=75-87 \%)$. 


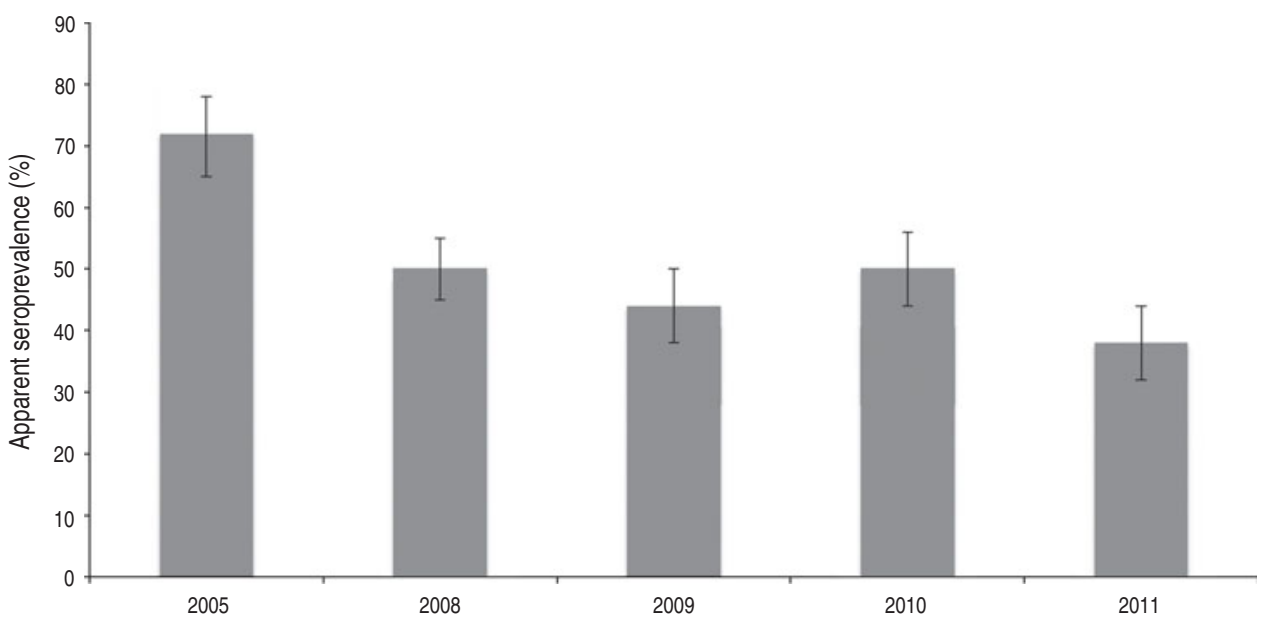

Fig. 3. Apparent seroprevalence (with $95 \%$ confidence intervals) in different years.

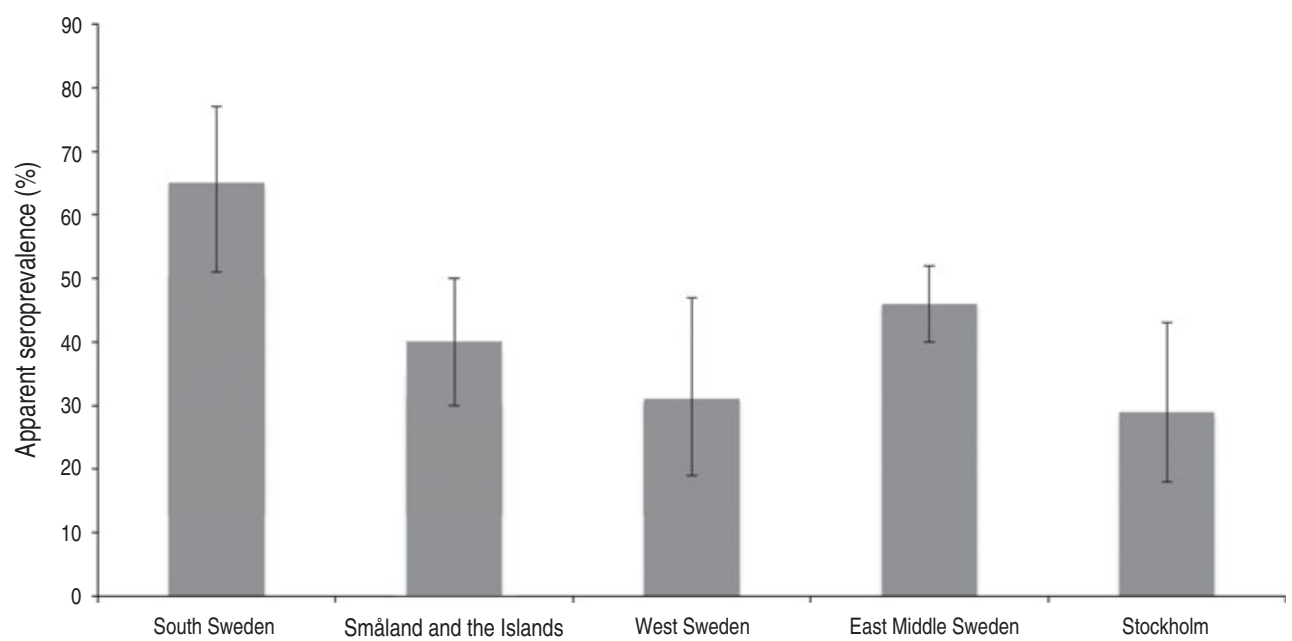

Fig. 4. Apparent seroprevalence (with 95\% confidence intervals) in different regions (Nomenclature of Territorial Units for Statistics level 2).

Lower and higher cut-offs displayed either too low $\mathrm{Sp}$ or too low Se for our purposes. Having a preference for a higher Se than Sp, the cut-off 0.39 was selected with $\mathrm{Se}=79 \%$ [95\% credibility interval (CrI) 60-95] and $\mathrm{Sp}=85 \%$ (95\% CrI 74-94). The cross-classified test results from the ELISA (cut-off 0.39) and the DAT on the 242 samples included in the latent class analysis are shown in Table 2. Including prior information in the model did not influence the posterior distribution of most parameters, except $\mathrm{Se}_{\mathrm{DAT}}$ and $\pi 2$. The results from the Se analysis are presented in Table 3.

\section{DISCUSSION}

This is the first study of $T$. gondii seroprevalence in wild boars in Sweden. Approximately half of the animals tested in this study were positive. Wild boar meat is becoming increasingly accessible in retail stores, and the modern consumer is not likely to be familiar with all possible health hazards associated with eating this meat undercooked. By analogy with the assumption that a large proportion of domestic pigs with antibodies to $T$. gondii also harbour viable tissue cysts [29], it is likely that people consuming wild boar meat in Sweden are at risk of contracting a $T$. gondii infection if the meat is ingested inadequately cooked.

Our finding of an age-dependent seroprevalence is in agreement with several other studies of $T$. gondii in wild boars [11, 12, 30]. However, not all studies have been able to confirm this [31]. Moreover, in a recent paper by Opsteegh and co-workers [10], it was 

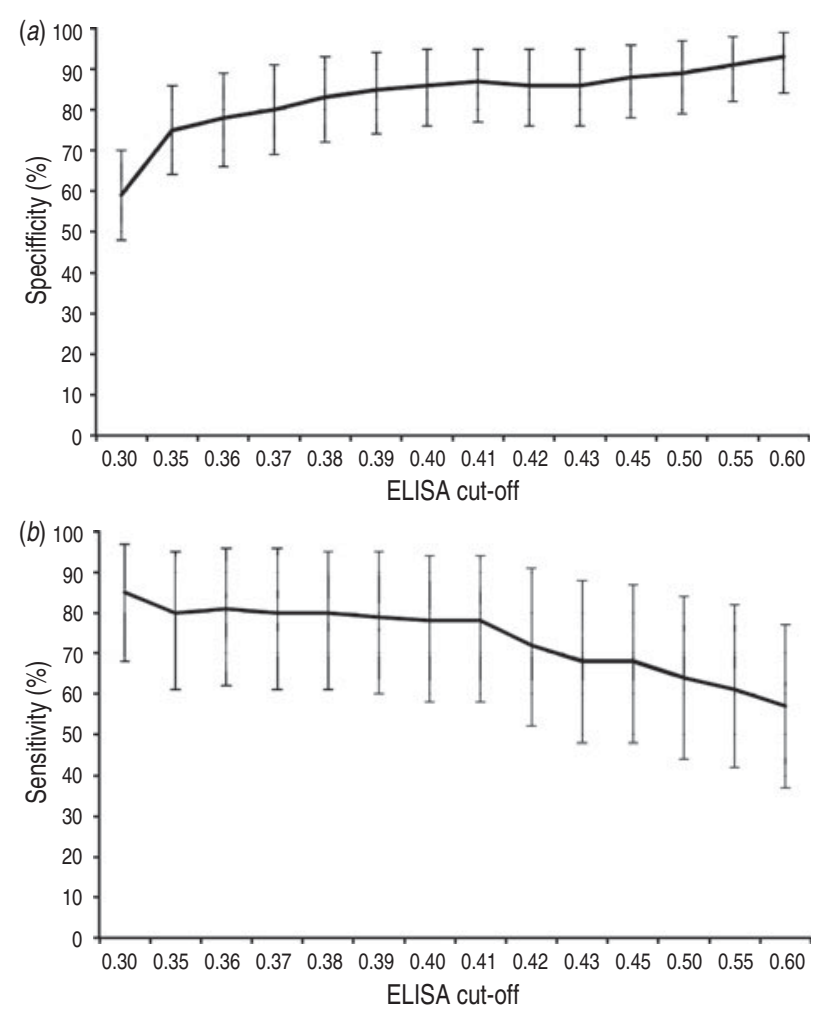

Fig. 5. Estimated (a) specificity and (b) sensitivity (including 95\% credibility interval) of the in-house ELISA at different cut-offs, based on the latent class analysis.

suggested that seropositive wild boars might seroconvert back to negative. This was based on the observation that seroprevalence increased with age, but reached a plateau at around age 10 months. Moreover, their data fitted best into a disease spread model with conversion back to susceptibility for previously infected animals. This hypothesis implies that infected wild boars might be able to reduce or eliminate the infection. Thus, further research addressing the relationship between seropositivity and presence of tissue cysts in wild boars, and possibly also in domestic pigs, is needed for a quantitative food safety risk assessment.

The apparent seroprevalence varied between years and it was significantly higher in the first year studied than in the later years. This could reflect decreased contamination with oocysts in the environment, which is believed to be the most important source of infection for wild boars. However, since the age of animals sampled in the earlier years (2005-2009) were not known, the result could be due to a higher proportion of older animals in those years. In addition, the seroprevalence for the years between 2005 and 2008 is missing, which possibly could have given a different picture if included. Lundén et al. [32] showed that the $T$. gondii prevalence varied between $10 \%$ and $45 \%$ in the same sheep flock when sampled twice a year for 6 years. This supports the hypothesis that environmental oocyst contamination is fluctuating at least locally, which could be reflected as a varying seroprevalence also in wild boars as in our study.

Due to the rapid expansion of the Swedish wild boar population during the study period and possible time-dependent differences in prevalence, we chose to compare regions only for the years 2010-2011. One region, South Sweden, stood out markedly with a significantly higher seroprevalence $(65 \%, 95 \%$ CI $51-77)$ than all other regions. This difference could not be explained by differences in age distributions since animals from the Småland and the Islands region, located just north of South Sweden, had a similar age distribution but a significantly lower prevalence (45\%, 95\% CI 38-53, $P<0 \cdot 05)$. A north-south gradient in $T$. gondii seroprevalence has previously been reported for example in moose and lynx in Sweden $[7,8]$ and in Finland $[33,34]$. These authors suggested that the most likely reason for the low seroprevalence in the far north is a low population density of domestic cats in these sparsely inhabited regions. Although the present study includes only the southern and central parts of Sweden, the recorded differences in seroprevalence might also be due to variations in human and cat populations. Other reasons could be meteorological factors such as precipitation [35] and winter temperatures [36], and geographical conditions such as altitude [37], which have been identified as risk factors for $T$. gondii infections in outdoor cats and wild boars. However, as the present study was based on a convenience sample, far-reaching conclusions concerning the differences found in seroprevalence between regions cannot be made.

A variety of $T$. gondii ELISA systems have been developed and used, sometimes without presenting estimations of diagnostic Se and Sp, making comparisons of test performance difficult. Moreover, commercial serological tests validated for use in defined host species, populations and sample materials are often used to screen entirely different ones, even though test evaluations have indicated that the same test can show different characteristics when applied to other species [16]. Thus, tests should be evaluated before use in new species and populations, and this should be considered when results are interpreted [38]. By estimating test performance for a series of 
Table 2. Cross-classified test results from the in-house ELISA (cut-off 0.39) and the DAT on the 242 samples included in the latent class analysis

\begin{tabular}{|c|c|c|c|c|c|c|}
\hline \multicolumn{4}{|c|}{ Population 1 ( $\leqslant 12$ months) } & \multicolumn{3}{|c|}{ Population 2 (>12 months) } \\
\hline & ELISA+ & ELISA- & Total & ELISA+ & ELISA- & Total \\
\hline DAT + & 29 & 15 & 44 & 37 & 8 & 45 \\
\hline DAT- & 13 & 86 & 99 & 10 & 44 & 54 \\
\hline Total & 42 & 101 & 143 & 47 & 52 & 99 \\
\hline
\end{tabular}

DAT, Direct agglutination test.

Table 3. Results of latent class sensitivity analysis, showing mean posterior estimate $195 \%$ credible interval) for all estimated parameters, when analysed using priors for DAT ( $\left.S e_{D A T} / S p_{D A T}\right)$, prevalences $(\pi 1 / \pi 2)$ and both

\begin{tabular}{|c|c|c|c|}
\hline \multirow{2}{*}{$\begin{array}{l}\text { Estimated } \\
\text { characteristic }\end{array}$} & \multicolumn{3}{|l|}{ Prior used } \\
\hline & $\mathrm{Se}_{\mathrm{DAT}} / \mathrm{Sp}_{\mathrm{DAT}}$ & $\pi 1 / \pi 2$ & Both \\
\hline $\mathrm{Se}_{\text {ELISA }}$ & 76 (55-94) & 76 (46-95) & $79(60-95)$ \\
\hline $\mathrm{Sp}_{\text {ELISA }}$ & $85(73-96)$ & $82(67-94)$ & $85(74-94)$ \\
\hline $\mathrm{Se}_{\mathrm{DAT}}$ & $79(61-93)$ & $77(43-98)$ & $82(69-93)$ \\
\hline $\mathrm{Sp}_{\mathrm{DAT}}$ & $87(78-95)$ & $83(66-96)$ & $87(80-94)$ \\
\hline$\pi 1$ & $27(12-43)$ & $26(19-34)$ & $27(20-34)$ \\
\hline$\pi 2$ & $50(33-72)$ & $43(33-54)$ & $44(34-59)$ \\
\hline
\end{tabular}

DAT, Direct agglutination test.

cut-off values we were able to select the most suitable cut-off for our prevalence study. At an OD cut-off of $0 \cdot 39$, the Se and $\mathrm{Sp}$ were estimated to $79 \%$ and $85 \%$, respectively, and this was regarded as adequate for the purposes of the survey. For diagnostic testing of individual animals, a higher $\mathrm{Se}$ and $\mathrm{Sp}$ would be preferable.

DAT is considered a reliable serological test for T. gondii $\mathrm{IgG}$ screening in many animal species [13]. The test is relatively often performed in new species (and animal populations) without a thorough evaluation of test characteristics. In the current study, DAT was evaluated at a single cut-off (1:20), because our primary goal was to retrieve estimates for the ELISA. The test characteristics derived for DAT at this cut-off were close to those obtained in similar studies of domestic pigs [39]. However, which cut-off to use for wild boar samples is a matter of debate. Richomme et al. [40] isolated viable parasites in 11/24 wild boars with end-point titres of 1:6 or 1:12, which may indicate recent infections with incipiently increasing antibody titres. This finding was interpreted as 1:6 being a biologically more relevant cut-off than 1:24 (i.e. many false-negative samples). On the other hand, Puvanesuaran et al. [41] found a perfect agreement between high serum titres ( $>1: 24)$ and presence of viable tissue cysts, whereas none of the samples with lower titres (1:6) were positive for tissue cysts. These results indicate that DAT characteristics need to be evaluated (for several cut-offs) when used on novel animal populations such as wild boars.

In the absence of a perfect reference test, the ELISA set up for this study was evaluated using a latent class approach. The Bayesian latent class process for test evaluation has been thoroughly described for chronic $T$. gondii infection in food animals by Gardner et al. [39] and has the advantage that estimates of diagnostic $\mathrm{Se}$ and $\mathrm{Sp}$ could be based on results from naturally infected animals, i.e. the target population of the investigation. In addition, previously collected information on test performances and population prevalences could be included in the model, giving more impartial and precise estimates. The model chosen was based on test results from two potentially dependent tests (ELISA and DAT) and two populations. Because many studies indicate a higher prevalence of $T$. gondii in older animals, subpopulations created here were based on age. However, one assumption in the calculations was that test $\mathrm{Se}$ and $\mathrm{Sp}$ were equal in both populations, and although age has been suggested as an appropriate factor in this model [39, 42], others have claimed that this is not advisable [43]. One argument is that older individuals may have been exposed to a greater variety of pathogens, which could lead to a difference in immune responses, and consequently, test performance. In our case, the probability of false-positive results may be slightly increased in the adult population (i.e. Sp is lower). If so, the true $\mathrm{Se}$ of the test is probably higher than estimated. 
This study has demonstrated a high seroprevalence of $T$. gondii in wild boars in Sweden and suggests that wild boar meat is a potential source of Toxoplasma infection to a growing group of consumers. Further studies are needed to establish risk factors and efforts must be made to inform the public how to prevent infection.

\section{ACKNOWLEDGEMENTS}

The authors thank Katarina Näslund and Göran Zakrisson for scientific advice, and Alison Burrells, Peter Lind and Per Wallgren for providing antigen and control sera. This work was funded by a grant from the Billström Foundation (2012). We also thank Lise-Lotte Fernström and Eva-Britt Jakubek for excellent technical assistance.

\section{DECLARATION OF INTEREST}

None.

\section{REFERENCES}

1. Petersen E, et al. What do we know about risk factors for infection in humans with Toxoplasma gondii and how can we prevent infections? Zoonoses and Public Health 2010; 57: 8-17.

2. Havelaar AH, et al. Disease burden of foodborne pathogens in the Netherlands, 2009. International Journal of Food Microbiology 2012; 156: 231-238.

3. Hoffmann S, Batz MB, Morris Jr JG. Annual cost of illness and quality-adjusted life year losses in the United States due to 14 foodborne pathogens. Journal of Food Protection 2012; 75: 1292-1302.

4. EFSA. Surveillance and monitoring of Toxoplasma in humans, food and animals, scientific opinion of the panel on biological hazards. EFSA Journal 2007; 583: $1-64$.

5. Gustafsson K, Uggla A. Serologic survey for Toxoplasma gondii infection in the brown hare (Lepus europaeus P.) in Sweden. Journal of Wildlife Diseases 1994; 30: 201-204.

6. Jakubek E-B, et al. Seroprevalences of Toxoplasma gondii and Neospora caninum in Swedish red foxes (Vulpes vulpes). Veterinary Parasitology 2001; 102: 167-172.

7. Malmsten J, Jakubek E-B, Björkman C. Prevalence of antibodies against Toxoplasma gondii and Neospora caninum in moose (Alces alces) and roe deer (Capreolus capreolus) in Sweden. Veterinary Parasitology 2011; 177: 275-280.

8. Ryser-Degiorgis M-P, et al. Serological survey of Toxoplasma gondii infection in free-ranging Eurasian lynx (Lynx lynx) from Sweden. Journal of Wildlife Diseases 2006; 42: 182-187.
9. Swedish Association for Hunting and Wildlife Management. Wildlife monitoring database (www.jagareforbundet.se/ Viltet/ViltVetande/Artpresentationer/Vildsvin). Accessed 11 June 2012.

10. Opsteegh M, et al. Age-related Toxoplasma gondii seroprevalence in Dutch wild boar inconsistent with lifelong persistence of antibodies. PLOS ONE 2011; 6: e 16240.

11. Jokelainen $\mathbf{P}$, et al. Farmed wild boars exposed to Toxoplasma gondii and Trichinella spp. Veterinary Parasitology 2012; 187: 323-327.

12. Deksne G, Kirjušina M. Seroprevalence of Toxoplasma gondii in domestic pigs (Sus scrofa domestica) and wild boars (Sus scrofa) in Latvia. Journal of Parasitology 2013; 99: 44-47.

13. Dubey JP. Toxoplasmosis of Animals and Humans, 2nd edn. Boca Raton, Florid: CRC Press, 2010, pp. 64.

14. Johnson WO, Gastwirth JL, Pearson LM. Screening without a 'Gold Standard': the Hui-Walter Paradigm Revisited. American Journal of Epidemiology 2001; 153: 921-924.

15. Frössling J, et al. Validation of a Neospora caninum iscom ELISA without a gold standard. Preventive Veterinary Medicine 2003; 57: 141-153.

16. Mainar-Jaime RC, Barberán M. Evaluation of the diagnostic accuracy of the modified agglutination test (MAT) and an indirect ELISA for the detection of serum antibodies against Toxoplasma gondii in sheep through Bayesian approaches. Veterinary Parasitology 2007; 148: 122-129.

17. Bengtsson B, et al. Surveillance of infectious diseases in animals and humans in Sweden 2011, National Veterinary Institute (SVA), Uppsala, Sweden (www. sva.se/upload/Redesign2011/Pdf/Om_SVA/publikationer/ Surveillance2011.pdf). Accessed 18 January 2013.

18. Thrusfield M, et al. WIN EPISCOPE $2 \cdot 0$ : improved epidemiological software for veterinary medicine. Veterinary Record 2001; 148: 567-572.

19. European Commission. European Commission Regulation (EC) No. 1059/2003 of the European Parliament and of the Council of 26 May 2003 on the establishment of a common classification of territorial units for statistics (NUTS). (http://eur-lex.europa.eu/ LexUriServ/LexUriServ.do?uri=OJ:L:2003:154:0001:0041: EN:PDF). Accessed 13 March 2013.

20. Opsteegh M, et al. Evaluation of ELISA test characteristics and estimation of Toxoplasma gondii seroprevalence in Dutch sheep using mixture models. Preventive Veterinary Medicine 2010; 96: 232-240.

21. Jakubek E-B, Lundén A, Uggla A. Seroprevalences of Toxoplasma gondii and Neospora sp. infections in Swedish horses. Veterinary Parasitology 2006; 138: 194-199.

22. Dubey JP, et al. Sensitivity and specificity of various serologic tests for the detection of Toxoplasma gondii infection in naturally infected sows. American Journal of Veterinary Research 1995; 56: 1030-1036.

23. Desmonts G, Remington JS. Direct agglutination test for diagnosis of Toxoplasma infection: method for increasing sensitivity and specificity. Journal of Clinical Microbiology 1980; 11: 562-568. 
24. Branscum AJ, Gardner IA, Johnson WO. Estimation of diagnostic-test sensitivity and specificity through Bayesian modeling. Preventive Veterinary Medicine 2005; 68: 145-163.

25. Spiegelhalter D, et al. OpenBUGS user manual, version 3.2.2. 2012. (http://www.openbugs.info/Manuals/Manual. html). Accessed 16 October 2012.

26. Brooks SP, Gelman A. General methods for monitoring convergence of iterative simulations. Journal of Computational and Graphical Statistics 1998; 7: $434-455$.

27. Youden WJ. Index for rating diagnostic tests. Cancer 1950; 3: 32-35.

28. R Core Team 2012. R: A language and environment for statistical computing. R Foundation for Statistical Computing, Vienna, Austria (http://www.R-project.org/).

29. Dubey JP, et al. High prevalence and genotypes of Toxoplasma gondii isolated from organic pigs in northern USA. Veterinary Parasitology 2012; 188: 14-18.

30. Berger-Schoch AE, et al. Toxoplasma gondii in Switzerland: a serosurvey based on meat juice analysis of slaughtered pigs, wild boar, sheep and cattle. Zoonoses and Public Health 2011; 58: 472-478.

31. Gauss CBL, et al. Seroprevalence of Toxoplasma gondii in wild pigs (Sus scrofa) from Spain. Veterinary Parasitology 2005; 131: 151-156.

32. Lundén A, Näsholm A, Uggla A. Long-term study of Toxoplasma gondii infection in a Swedish sheep flock. Acta Veterinaria Scandinavica 1994; 35: 273-281.

33. Jokelainen $\mathbf{P}$, et al. Toxoplasma gondii in wild cervids and sheep in Finland: north-south gradient in seroprevalence. Veterinary Parasitology 2010; 171: 331-336.

34. Jokelainen P, et al. Free-ranging Eurasian lynx (Lynx lynx) as host of Toxoplasma gondii in Finland. Journal of Wildlife Diseases 2013; 49: 527-534.
35. Afonso E, Thulliez P, Gilot-Fromont E. Local meteorological conditions, dynamics of seroconversion to Toxoplasma gondii in cats (Felis catus) and oocyst burden in a rural environment. Epidemiology and Infection 2010; 138: 1105-1113.

36. Beral M, et al. Environmental factors associated with the seroprevalence of Toxoplasma gondii in wild boars (Sus scrofa), France. EcoHealth 2012; 9: 303-309.

37. Richomme C, et al. Seroprevalence and factors associated with Toxoplasma gondii infection in wild boar (Sus scrofa) in a Mediterranean island. Epidemiology and Infection 2010; 138: 1257-1266.

38. Gardner IA. An Epidemiologic critique of current microbial risk assessment practices: the importance of prevalence and test accuracy data. Journal of Food Protection 2004; 67: 2000-2007.

39. Gardner IA, Greiner M, Dubey JP. Statistical evaluation of test accuracy studies for Toxoplasma gondii in food animal intermediate hosts. Zoonoses Public Health 2010; 57: 82-94.

40. Richomme C, et al. Genetic characterization of Toxoplasma gondii from wild boar (Sus scrofa) in France. Veterinary Parasitology 2009; 164: 296-300.

41. Puvanesuaran VR, Noordin R, Balakrishnan V. Genotyping of Toxoplasma gondii isolates from wild boars in Peninsular Malaysia. PLoS ONE 2013; 8: e61730.

42. Hui SL, Walter SD. Estimating the error rates of diagnostic tests. Biometrics 1980; 36: 167-171.

43. Toft N, Jørgensen E, Højsgaard S. Diagnosing diagnostic tests: evaluating the assumptions underlying the estimation of sensitivity and specificity in the absence of a gold standard. Preventive Veterinary Medicine 2005; 68: 19-33. 